\title{
Establishment of a specimen panel for the decentralised technical evaluation of the sensitivity of 31 rapid diagnostic tests for SARS-CoV-2 antigen, Germany, September 2020 to April 2021
}

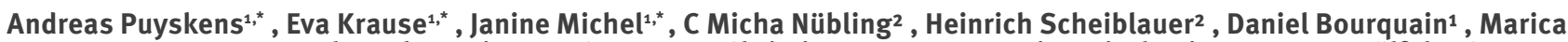
Grossegesse $^{1}$, Roman Valusenko ${ }^{1}$, Victor M Corman ${ }^{3,4}$, Christian Drosten ${ }^{3}$, Katrin Zwirglmaier ${ }^{5}$, Roman Wölfel ${ }^{5}$, Constanze Lange $^{6}$, Jan Kramer 6 , Johannes Friesen ${ }^{7}$, Ralf Ignatius ${ }^{7}$, Michael Müller ${ }^{7}$, Jonas Schmidt-Chanasit ${ }^{8}$, Petra Emmerich ${ }^{8,9}$, Lars Schaade ${ }^{1}$, Andreas Nitsche ${ }^{1}$

1. Robert Koch Institute, Highly Pathogenic Viruses, Centre for Biological Threats and Special Pathogens, WHO Reference Laboratory for SARS-CoV-2 and WHO Collaborating Centre for Emerging Infections and Biological Threats, Robert Koch Institute, Berlin, Germany

2. Testing Laboratory for In-vitro Diagnostic Medical Devices, Paul-Ehrlich-Institute, Langen, Germany

3. Charité - Universitätsmedizin Berlin, Institute of Virology and German Centre for Infection Research (DZIF), Associated Partner Site, Berlin, Germany

4. Labor Berlin, Charité - Vivantes GmbH, Berlin, Germany

5. Bundeswehr Institute of Microbiology and German Centre for Infection Research (DZIF), Partner Site Munich, Munich, Germany

6. LADR Central Laboratory Dr. Kramer \& Colleagues, Geesthacht, Germany

7. MVZ Labor $28 \mathrm{GmbH}$, Berlin, Germany

8. Bernhard Nocht Institute for Tropical Medicine, Arbovirology Department, Hamburg, Germany

9. Department of Tropical Medicine and Infectious Diseases, Center of Internal Medicine II, University of Rostock, Rostock, Germany

* These authors contributed equally to this work and share first authorship.

Correspondence: Andreas Nitsche (NitscheA@rki.de)

Puyskens Andreas, Krause Eva, Michel Janine, Nübling C Micha, Scheiblauer Heinrich, Bourquain Daniel, Grossegesse Marica, Valusenko Roman, Corman Victor M, Drosten Christian, Zwirglmaier Katrin, Wölfel Roman, Lange Constanze, Kramer Jan, Friesen Johannes, Ignatius Ralf, Müller Michael, Schmidt-Chanasit Jonas, Emmerich Petra, Schaade Lars, Nitsche Andreas. Establishment of a specimen panel for the decentralised technical evaluation of the sensitivity of 31 rapid

diagnostic tests for SARS-CoV-2 antigen, Germany, September 2020 to April 2021. Euro Surveill. 2021;26(44):pii=2100442. https://doi.org/10.2807/1560-7917.

ES.2021.26.44.2100442

Article submitted on 08 May 2021 / accepted on 10 Sep 2021 / published on 04 Nov 2021

Introduction: The detection of SARS-CoV-2 with rapid diagnostic tests (RDT) has become an important tool to identify infected people and break infection chains. These RDT are usually based on antigen detection in a lateral flow approach. Aim: We aimed to establish a comprehensive specimen panel for the decentralised technical evaluation of SARS-CoV-2 antigen rapid diagnostic tests. Methods: While for PCR diagnostics the validation of a PCR assay is well established, there is no common validation strategy for antigen tests, including RDT. In this proof-of-principle study we present the establishment of a panel of 50 pooled clinical specimens that cover a SARS-CoV-2 concentration range from $1.1 \times 10^{9}$ to 420 genome copies per $\mathrm{mL}$ of specimen. The panel was used to evaluate 31 RDT in up to six laboratories. Results: Our results show that there is considerable variation in the detection limits and the clinical sensitivity of different RDT. We show that the best RDT can be applied to reliably identify infectious individuals who present with SARSCoV-2 loads down to $10^{6}$ genome copies per $\mathrm{mL}$ of specimen. For the identification of infected individuals with SARS-CoV-2 loads corresponding to less than
$10^{6}$ genome copies per $\mathrm{mL}$, only three RDT showed a clinical sensitivity of more than $60 \%$.

Conclusions: Sensitive RDT can be applied to identify infectious individuals with high viral loads but not to identify all infected individuals.

\section{Introduction}

PCR-based diagnostics of severe acute respiratory syndrome coronavirus 2 (SARS-CoV-2) is a well-established method with numerous commercially available kits and in-house assays published over the last months [1]. Although it is beyond question that in particular realtime PCR provides an unrivalled degree of analytical sensitivity and reproducibility, there are some obvious limitations [2]. Above all, PCR-based diagnostics requires a functioning laboratory infrastructure and skilled personnel. The PCR reaction itself requires only around $2 \mathrm{~h}$, including pre- and post-analytical steps; however, time to result in high-throughput mode is usually $24 \mathrm{~h}$ or more.

The need for faster and simpler approaches to diagnose a SARS-CoV-2 infection is therefore evident as well as the need for on-site tests and for diagnostics in 
TABLE 1

Characteristics of the 50 pools of SARS-CoV-2 clinical specimens constituting Panel 1V1 and Panel 1V2, Germany, September 2020-April 2021

\begin{tabular}{|c|c|c|c|c|c|}
\hline \multicolumn{3}{|c|}{ Panel $1 V_{1}$} & \multicolumn{3}{|c|}{ Panel $1 V_{2}$} \\
\hline Pool number & $\mathrm{Cq} / 5 \mu \mathrm{L}$ of RNA & RNA subjected to test & Pool number & $\mathrm{Cq} / 5 \mu \mathrm{L}$ of RNA & RNA subjected to test \\
\hline 1 & 17.55 & $1.1 \times 10^{7}$ & 1 & $17 \cdot 31$ & $1.31 \times 10^{7}$ \\
\hline 2 & 20.54 & $1.4 \times 10^{6}$ & 2 & 19.08 & $3.87 \times 10^{6}$ \\
\hline 3 & 20.38 & $1.6 \times 10^{6}$ & 3 & 19.62 & $2.67 \times 10^{6}$ \\
\hline 4 & 20.98 & $1.0 \times 10^{6}$ & 4 & 20.61 & $1.35 \times 10^{6}$ \\
\hline 5 & 20.28 & $1.7 \times 10^{6}$ & 5 & 20.60 & $1.36 \times 10^{6}$ \\
\hline 6 & 20.20 & $1.8 \times 10^{6}$ & 6 & 21.21 & $8.96 \times 10^{5}$ \\
\hline 7 & 21.71 & $6.4 \times 10^{5}$ & 7 & 22.15 & $4.70 \times 10^{5}$ \\
\hline 8 & 21.95 & $5.4 \times 10^{5}$ & 8 & 22.32 & $4.18 \times 10^{5}$ \\
\hline 9 & 22.14 & $4.7 \times 10^{5}$ & 9 & 23.13 & $2.39 \times 10^{5}$ \\
\hline 10 & 22.88 & $2.8 \times 10^{5}$ & 10 & 23.21 & $2.27 \times 10^{5}$ \\
\hline 11 & 22.34 & $4.1 \times 10^{5}$ & 11 & 23.13 & $2.27 \times 10^{5}$ \\
\hline 12 & 21.82 & $5.9 \times 10^{5}$ & 12 & 22.12 & $4.79 \times 10^{5}$ \\
\hline 13 & $23 \cdot 32$ & $2.1 \times 10^{5}$ & 13 & 25.29 & $5.42 \times 10^{4}$ \\
\hline 14 & 24.28 & $1.1 \times 10^{5}$ & 14 & 24.97 & $6.76 \times 10^{4}$ \\
\hline 15 & 24.14 & $1.2 \times 10^{5}$ & 15 & 24.38 & $1.01 \times 10^{5}$ \\
\hline 16 & 22.55 & $3.6 \times 10^{5}$ & 16 & 22.88 & $2.84 \times 10^{5}$ \\
\hline 17 & 24.00 & $1.3 \times 10^{5}$ & 17 & 24.81 & $7.54 \times 10^{4}$ \\
\hline 18 & $25 \cdot 30$ & $5.4 \times 10^{4}$ & 18 & 28.33 & $6.71 \times 10^{3}$ \\
\hline 19 & $25 \cdot 50$ & $4.7 \times 10^{4}$ & 19 & 25.45 & $2.39 \times 10^{5}$ \\
\hline 20 & 26.27 & $2.8 \times 10^{4}$ & 20 & 29.46 & $2.27 \times 10^{5}$ \\
\hline 21 & 25.54 & $4.6 \times 10^{4}$ & 21 & 25.95 & $2.27 \times 10^{5}$ \\
\hline 22 & 25.87 & $3.7 \times 10^{4}$ & 22 & 27.42 & $4.79 \times 10^{5}$ \\
\hline 23 & 24.04 & $1.3 \times 10^{5}$ & 23 & 24.45 & $5.42 \times 10^{4}$ \\
\hline 24 & 25.24 & $5.6 \times 10^{4}$ & 24 & 25.20 & $6.76 \times 10^{4}$ \\
\hline 25 & 29.70 & $2.6 \times 10^{3}$ & 25 & 25.07 & $1.01 \times 10^{5}$ \\
\hline 26 & 25.47 & $4.8 \times 10^{4}$ & 26 & 26.32 & $2.84 \times 10^{5}$ \\
\hline 27 & 25.14 & $6.0 \times 10^{4}$ & 27 & 26.12 & $7.54 \times 10^{4}$ \\
\hline 28 & 27.14 & $1.5 \times 10^{4}$ & 28 & $27 \cdot 41$ & $6.71 \times 10^{3}$ \\
\hline 29 & 27.15 & $1.5 \times 10^{4}$ & 29 & 27.34 & $1.33 \times 10^{4}$ \\
\hline 30 & 28.86 & $4.7 \times 10^{3}$ & 30 & 27.24 & $1.42 \times 10^{4}$ \\
\hline 31 & 25.27 & $5.5 \times 10^{4}$ & 31 & 26.24 & $1.42 \times 10^{4}$ \\
\hline 32 & 26.44 & $2.5 \times 10^{4}$ & 32 & 26.64 & $2.14 \times 10^{4}$ \\
\hline 33 & 28.96 & $4.4 \times 10^{3}$ & 33 & 28.92 & $4.47 \times 10^{3}$ \\
\hline 34 & 27.89 & $9.1 \times 10^{3}$ & 34 & 27.82 & $9.53 \times 10^{3}$ \\
\hline 35 & 27.04 & $1.6 \times 10^{4}$ & 35 & 26.66 & $2.12 \times 10^{4}$ \\
\hline 36 & 28.13 & $7.7 \times 10^{3}$ & 36 & 27.05 & $1.62 \times 10^{4}$ \\
\hline 37 & 30.54 & $1.5 \times 10^{3}$ & 37 & 30.13 & $1.95 \times 10^{3}$ \\
\hline 38 & 28.14 & $7.6 \times 10^{3}$ & 38 & 29.36 & $3.31 \times 10^{3}$ \\
\hline 39 & 29.76 & $2.5 \times 10^{3}$ & 39 & 30.12 & $1.96 \times 10^{3}$ \\
\hline 40 & 27.65 & $1.1 \times 10^{4}$ & 40 & 28.19 & $7.39 \times 10^{3}$ \\
\hline 41 & 30.13 & $1.9 \times 10^{3}$ & 41 & 30.14 & $7.39 \times 10^{3}$ \\
\hline 42 & 28.43 & $6.2 \times 10^{3}$ & 42 & 29.48 & $3.04 \times 10^{3}$ \\
\hline 43 & 31.05 & $1.0 \times 10^{3}$ & 43 & 31.61 & $7.04 \times 10^{2}$ \\
\hline 44 & 29.24 & $3.6 \times 10^{3}$ & 44 & $29 \cdot 51$ & $2.98 \times 10^{3}$ \\
\hline 45 & 30.10 & $2.0 \times 10^{3}$ & 45 & 31.19 & $9.40 \times 10^{2}$ \\
\hline 46 & 31.54 & $7.4 \times 10^{2}$ & 46 & 31.34 & $8.48 \times 10^{2}$ \\
\hline 47 & 35.19 & $6.0 \times 10^{1}$ & 47 & 34.55 & $9.34 \times 10^{1}$ \\
\hline 48 & 32.06 & $5.2 \times 10^{2}$ & 48 & 31.19 & $9.40 \times 10^{2}$ \\
\hline 49 & 35.22 & $5.9 \times 10^{1}$ & 49 & 36.04 & $3.35 \times 10^{1}$ \\
\hline 50 & 36.36 & $2.7 \times 10^{1}$ & 50 & 35.83 & $3.87 \times 10^{1}$ \\
\hline
\end{tabular}

Cq: quantification cycle; SARS-CoV-2: severe acute respiratory syndrome coronavirus 2.

Cq values in bold indicate that SARS-CoV-2 could be propagated in cell culture. 
regions with lower standards of laboratory infrastructure [3-5]. One promising technology to detect SARSCoV-2-specific proteins in respiratory secretions are lateral flow immunoassays which operate within less than $30 \mathrm{~min}$, so-called rapid diagnostic tests (RDT) [6]. The trade-off for a simple and quick diagnostic test is an often considerably lower analytical sensitivity and specificity compared with nucleic acid amplification techniques such as PCR $[7,8]$.

Besides the traditional RDT whose read-out is performed visually, there are assays that utilise readers for the identification of positive signals. These readers can provide a better sensitivity, reproducibility and objectivity, in particular with fluorescence-based formats; however, the mobility of testing as well as the parallel testing of many specimens can be negatively affected.

Here we describe the decentralised evaluation of the sensitivity of 31 RDT using an identical panel of 50 clinical specimens analysed by up to six of seven participating German laboratories, namely those at the Robert Koch Institute, the Paul Ehrlich Institute, the Charité, as well as the Bundeswehr Institute of Microbiology, the LADR Central Laboratory Dr. Kramer \& Colleagues, the MVZ Labor28 GmbH and at the Bernhard Nocht Institute for Tropical Medicine. With this approach, we generated at least two independent results per RDT and hence addressed inter-laboratory variations that have to be considered when RDT are performed in different locations by different persons.

\section{Methods}

\section{Evaluation panel}

To enable the systematic and comparable decentralised evaluation of numerous RDT, we compiled a panel of 50 samples by pooling a total of around 500 upper respiratory specimens from symptomatic patients, collected between March and September 2020 (Panel 1V1). For pooling, we mainly used dry swabs resuspended in phosphate-buffered saline (PBS) and a small number of swabs obtained in viral transport medium, resulting in a final concentration of viral transport medium of $\leq 20 \%$ in each pool, $\leq 10 \%$ in 38 pools and $0 \%$ in 25 pools.

Subsequently, up to 10 respiratory specimens obtained for routine diagnostics, with different virus loads as determined by real-time PCR, were pooled and diluted to a defined RNA load in a background of negative swabs in PBS. Pools were frozen at $-80^{\circ} \mathrm{C}$. Realtime PCR was applied to determine the RNA load per pool [9]. In vitro RNA (provided by the World Health Organization (WHO)) as well as the quantitative reference material provided by INSTAND were used for quantification (https://www.instand-ev.de). Finally, the panel covered a range of SARS-CoV-2 RNA from $1.1 \times 10^{9}$ genomes per $\mathrm{mL}$ down to 420 genomes per $\mathrm{mL}$. When Panel ${ }_{1} \mathrm{~V}_{1}$ was used up, new pools were generated by diluting the same samples as for Panel 1V1 (except for four pools 1-4 that had to be constituted from new clinical specimens collected between October 2020 and January 2021), resulting in comparable virus loads as determined by real-time PCR, and that panel was labelled Panel $1 V_{2}$. In addition, we compared Panel $1 V_{1}$ with Panel $1 V_{2}$ in RDT \# 3 and RDT \#31 and the results were identical. Panel $1 V_{1}$ and Panel $1 V_{2}$ were later used to routinely evaluate the sensitivity of 122 RDT as described in the tandem publication by Scheiblauer et al. [10]. Here, we highlight the results of 31 of those RDT to give a more comprehensive insight into the performance of the evaluation panel. As negative control, we pooled respiratory specimens obtained by swabbing SARS-CoV-2-negative individuals.

Previous studies have revealed that a minimal RNA genome copy number of $10^{6}$ genome copies per $\mathrm{mL}$ of specimen represents the amount of infectious virus particles required for successful virus propagation in cell culture [9,11-14]. To correlate the pools to potential infectivity in a specimen, we propagated the pools with $\geq 10^{6}$ genome copies per $\mathrm{mL}$, corresponding to a quantification cycle $(\mathrm{Cq})$ value 25 , in cell culture. Confirmation of replication-competent SARS-CoV-2 was achieved by inoculation of VeroE6 cells with the respective pools. Pools containing infectious SARSCoV-2 were subsequently titrated on VeroE6 cells. However, even if pools containing higher amounts of SARS-CoV-2 RNA generally showed higher titres than those with lower genome numbers, we observed no substantial correlation between the genome load and the titre (data not shown).

The specifications of the 50 pools are listed in Table 1; pools allowing SARS-CoV-2 propagation are marked in bold.

\section{Selection of rapid diagnostic tests}

The RDT included in this study were selected at random according to availability at the time of the study (Table 2). No technical assumptions were made in the RDT selection process.

\section{Rapid diagnostic test procedure}

Except when RDT were evaluated without the included swabs, RDT were used according to the manufacturers' instructions. In brief, $50 \mu \mathrm{L}$ of a pool were either directly added to the provided test buffer in volumes recommended by the manufacturer, or the swab included in the RDT kit was used to absorb the $50 \mu \mathrm{L}$ and then subjected to the RDT procedure as recommended by the manufacturer. Results obtained by visual examination of the test device in different laboratories were categorised as 'positive' or 'negative' and subjected to statistical analysis by using the GraphPad Prism software as indicated in the Results section. Results were only accepted when the control band was positive, which was the case in more than $99 \%$ of the tested RDT. 
TABLE 2

SARS-CoV-2 rapid antigen tests evaluated in this study, Germany, September 2020-April $2021(\mathrm{n}=31)$

\begin{tabular}{|c|c|c|c|c|}
\hline Number & Manufacturer/distributer (town, country) & Name & $\begin{array}{l}\text { Number of } \\
\text { evaluating } \\
\text { laboratories }\end{array}$ & $\begin{array}{l}\text { Target } \\
\text { antigen }\end{array}$ \\
\hline \multicolumn{5}{|c|}{ Classical point-of-care tests } \\
\hline 1 & $\begin{array}{l}\text { SD BIOSENSOR (Suwon-si, South Korea), distributed by } \\
\text { Roche Diagnostics GmbH (Mannheim, Germany) }\end{array}$ & SARS-CoV-2 Rapid Antigen Test & 6 & N.s. \\
\hline 2 & Abbott (Jena, Germany) & $\begin{array}{l}\text { Panbio COVID-19 Ag Rapid Test Device } \\
\text { (Nasopharyngeal) }\end{array}$ & 5 & N.s. \\
\hline 3 & R-Biopharm AG (Darmstadt, Germany) & RIDA QUICK SARS-CoV-2 Antigen & 3 & $\mathrm{~N}$ protein \\
\hline 4 & servoprax GmbH (Wesel, Germany) & Cleartest & 2 & N.s. \\
\hline 5 & $\begin{array}{c}\text { Jiangsu Changfeng Medical Industry Co. (Touqiao Town, } \\
\text { China), Ltd., distributed by nal von minden GmbH (Moers, } \\
\text { Germany) }\end{array}$ & Dedicio COVID-19 Ag plus Test & 3 & $\mathrm{~N}$ protein \\
\hline 6 & nal von minden $\mathrm{GmbH}$ & NADAL COVID-19 Ag Test & 3 & $\mathrm{~N}$ protein \\
\hline 7 & $\begin{array}{c}\text { RapiGEN Inc. (Gunpo-si, South Korea), distributed by Weko } \\
\text { Pharma (Pellingen, Germany) }\end{array}$ & BIOCREDIT COVID-19 Ag & 3 & N.s. \\
\hline 8 & SD BIOSENSOR & STANDARD Q COVID-19 Ag Test & 2 & N.s. \\
\hline 9 & Biosynex Swiss SA (Freiburg, Switzerland) & BIOSYNEX COVID-19 Ag BSS & 2 & $\mathrm{~N}$ protein \\
\hline 10 & MEDsan GmbH (Hamburg, Germany) & MEDsan SARS-CoV-2 Antigen Rapid Test & 2 & N.s. \\
\hline 11 & $\begin{array}{l}\text { BIONOTE (Hwaseong-si, South Korea), distributed by } \\
\text { concile (Freiburg, Germany) }\end{array}$ & NowCheck COVID-19 Ag Test & 3 & N.s. \\
\hline 12 & Zhejiang Orient Gene Biotech Co., Ltd (Huzhou, China) & COVID-19 Rapid Ag Test Cassette & 2 & $\mathrm{~N}$ protein \\
\hline 13 & Fujirebio Inc. (Tokyo, Japan) & ESPLINE SARS-CoV-2 & 2 & N.s. \\
\hline 14 & Coris BioConcept (Gembloux, Belgium) & COVID-19 Ag Respi-Strip & 2 & $\mathrm{~N}$ protein \\
\hline 15 & Healgen Scientific LLC (Houston TX, US) & $\begin{array}{l}\text { Healgen Coronavirus Ag Rapid Test Cassette } \\
\text { (Swab) }\end{array}$ & 2 & $\mathrm{~N}$ protein \\
\hline 16 & Günter Keul GmbH (Steinfurt, Germany) & Keul-o-test & 2 & N.s. \\
\hline 17 & Acro Biotech. Inc. (Rancho Cucamonga CA, US) & $\begin{array}{c}\text { Acro Rapid Test COVID-19 Antigen Rapid Test } \\
\text { (Nasopharyngeal Swab) }\end{array}$ & 2 & $\mathrm{~N}$ protein \\
\hline 18 & MEXACARE GmbH (Heidelberg, Germany) & $\begin{array}{l}\text { COVID-19 Antigen Schnelltest } \\
\text { (Nasen-Rachenabstrich) }\end{array}$ & 2 & $\mathrm{~N}$ protein \\
\hline 19 & Beijing Beier Bioengineering Co., Ltd. (Beijing, China) & COVID-19 Antigen Rapid Test Kit & 2 & $\mathrm{~N}$ protein \\
\hline 20 & möLaboratory GmbH (Langenfeld, Germany) & $\begin{array}{l}\text { mö-screen Testkit Corona Antigen } \\
\text { Nasenabstrich }\end{array}$ & 2 & $\mathrm{~N}$ protein \\
\hline 21 & $\begin{array}{l}\text { Xiamen Biotime Biotechnology Co., Ltd. (Fujian, China), } \\
\text { distributed by MEDICE Arzneimittel Pütter GmbH and Co. } \\
\text { KG (Iserlohn, Germany) }\end{array}$ & Medicovid SARS-CoV-2 AG Antigen Schnelltest & 2 & $\mathrm{~N}$ protein \\
\hline 22 & $\begin{array}{l}\text { Green Cross Medical Science Corp. (Eumseong-gun, South } \\
\text { Korea) }\end{array}$ & GENEDIA COVID-19 Ag & 2 & N.s. \\
\hline 23 & $\begin{array}{c}\text { Guangdong Wesail Biotech Co., Ltd. (Dongguan, China), } \\
\text { distributed by Bio-Gram Diagnostics GmbH (Ludwigshafen } \\
\text { am Rhein, Germany) }\end{array}$ & COVID-19 Antigen Test Kit & 2 & $\mathrm{~N}$ protein \\
\hline 24 & $\begin{array}{c}\text { Joinstar Biomedical Technology Co., Ltd. (Hangzhou, } \\
\text { China), distributed by care impuls Vertriebs GmbH (Ried, } \\
\text { Germany) }\end{array}$ & COVID-19 Antigen Schnelltest (Colloidal Gold) & 2 & $\mathrm{~N}$ protein \\
\hline 25 & $\begin{array}{c}\text { Hangzhou Realy Tech Co. Ltd. (Hangzhou, China), } \\
\text { distributed by TREKSTOR GmbH (Bensheim, Germany) }\end{array}$ & $\begin{array}{l}\text { blnk COVID-19 Antigen Rapid Test } \\
\text { (Nasopharyngeal Swab) }\end{array}$ & 2 & N.s. \\
\hline 26 & $\begin{array}{l}\text { Hangzhou Laihe Biotech Co., Ltd. (Hangzhou, China), } \\
\text { distributed by Lissner Qi GmbH (Hamburg, Germany) }\end{array}$ & Novel Coronavirus (COVID-19) Antigen Test Kit & 2 & $\mathrm{~N}$ protein \\
\hline 27 & Koch Biotechnology (Beijing) Co., Ltd. (Beijing, China) & COVID-19 Antigen Rapid Test Strip & 2 & S protein \\
\hline \multicolumn{5}{|c|}{ Rapid diagnostic tests requiring a read-out device } \\
\hline 28 & SD Biosensor & STANDARD F COVID-19 Ag FIA & 4 & $\mathrm{~N}$ protein \\
\hline 29 & Quidel Corporation (San Diego CA, US) & Sofia SARS Antigen FIA & 3 & $\mathrm{~N}$ protein \\
\hline 30 & Schebo Biotech AG (Gießen, Germany) & ScheBo SARS-CoV-2 Quick Antigen & 3 & $\mathrm{~N}$ protein \\
\hline 31 & Beijing Wantai Biological Pharmacy (Beijing, China) & Wantai SARS-CoV-2 Antigen Rapid Test (FIA) & 2 & $\mathrm{~N}$ protein \\
\hline
\end{tabular}

N: nucleocapsid; N.s.: not specified; S: spike. 
Ethical statement

The study obtained ethical approval by the Berliner Ärztekammer (Berlin Chamber of Physicians, Eth 20/40).

\section{Results}

Establishing the evaluation panel

Before distributing the panel, we compared the detectability of specimens in two RDT (\#2, \#3) before and after freezing at $-40^{\circ} \mathrm{C}$ and did not observe any considerable differences in the virus concentration range close to the detection limit<20,000 RNA copies per $\mathrm{mL}$ (data not shown). Furthermore, to test whether pooling and freezing had an impact on the detectability of specimens, we compared 40-44 fresh clinical specimens, representing a $\mathrm{Cq}$ value range from 20 to $35\left(1.8 \times 10^{7}\right.$ to $\left.7.0 \times 10^{3}\right)$ genome copies per $\mathrm{mL}$, with 32 pools of Panel $1 \mathrm{~V}_{1}$, covering a comparable $\mathrm{Cq}$ value range using 10 RDT (\#2, \#3, \#7, \#8, \#10, \#11, \#14, \#21, \#28 and \#31). In none of the RDT tested did we observe a considerable discrepancy between the detectability of fresh specimens and pools with a comparable $\mathrm{Cq}$ value (data summarised in Supplementary Figure $\mathrm{S}_{1}$ ).

Since some of the swabs had been transported to the laboratory in viral transport medium, the final concentration of viral transport medium in pools was $\leq 20 \%$ $\mathrm{v} / \mathrm{v}$ for each pool, $\leq 10 \% \mathrm{v} / \mathrm{v}$ for 38 pools and $0 \%$ for 25 pools. Ten RDT were randomly selected to calculate whether viral transport medium had an impact on RDT sensitivity. Supplementary Figure S2 illustrates the results for one of the tested RDT; binary logistic regression revealed no difference in the detection probability of pools containing varying amounts of medium. Ultimately, we did not observe an influence of viral transport medium in the evaluation panel on RDT sensitivity.

\section{Analytical sensitivity of rapid diagnostic tests}

All samples were stored at $-40^{\circ} \mathrm{C}$ before shipment and were transferred to dry ice for shipping. The panels were shipped to the participating laboratories as two vials à $500 \mu \mathrm{L}$ per sample. Upon arrival, samples were thawed, mixed and aliquoted in $50 \mu \mathrm{L}$ aliquots to be used for testing. After thawing, aliquots were used immediately to allow maximum comparability between laboratories and test days. Figure 1 summarises the workflow as recommended to the participating laboratories. The results were assessed visually by experienced laboratory personnel.

Figure 2 presents the sensitivity results for the 27 RDT that could be analysed visually (RDT \#1-27) and the results for the four RDT that needed a device for readout (RDT \#28-31). Based on binary logistic regression of all results obtained from the different participating laboratories, the $50 \%$ probability to detect a certain genome load was calculated as a marker for the detection limit of an RDT. Seven of 31 RDT showed a $50 \%$ detection probability for genome loads higher than $10^{6}$ genome copies per $\mathrm{mL}, 24$ had a $50 \%$ detection probability of less than $10^{6}$ genome copies per $\mathrm{mL}$, while 15 had a $50 \%$ detection probability of less than $10^{5}$ genome copies per $\mathrm{mL}$. The most sensitive RDT detected around 75,000 genome copies per $\mathrm{mL}$ with a probability of $90 \%$, while the least sensitive RDT showed a $90 \%$ detection probability for $2.3 \times 10^{7}$ genomes per $\mathrm{mL}$.

Figure 2 also shows that using the RDT-specific swabs to absorb the pool material before testing can lead to a loss of analytical sensitivity at a factor of 10 to 50, although some of the RDT showed only small differences between direct application of the pool and swab usage. This indicates varying efficiency of the absorption and release characteristics for SARS-CoV-2 particles from the swabs.

\section{Clinical sensitivity of rapid diagnostic tests}

To determine the clinical sensitivity of an RDT, results from different laboratories were merged and categorised according to the genome load, e.g. the Cq value obtained for each pool by real-time PCR [9]: Cq<25 ( $10^{6}$ genome copies per $\mathrm{mL}$ ), $25 \leq \mathrm{Cq}<30$ and $\mathrm{Cq} \geq 30$. Sensitivities of each RDT to identify pools correctly were calculated for each Cq category.

According to previous studies, Cq<25 corresponds to an increased probability of a specimen to successfully propagate SARS-CoV-2 in cell culture $[9,11,12]$. This was confirmed for nine of 18 pools with Cq values $<25$ for Panel $1 V_{1}$ and five of 17 pools for Panel $1 V_{2}$ (see bold entries in Table 1 ). Pools with a $\mathrm{Cq} \geq 30$ were highly unlikely to contain virus amounts high enough to grow in cell culture. Specimens with $\mathrm{Cq}$ values between 25 and 30 very rarely propagated virus in cell culture.

Figure 3 summarises the sensitivities for the 31 RDT. Figure 3A shows the sensitivity for all 50 SARSCoV-2-positive pools of the panel. Results are further categorised according to the different virus loads represented by a pool. Figure $3 \mathrm{~B}$ depicts pools with $\mathrm{Cq}<25\left(\mathrm{n}=18\right.$ Panel $1 \mathrm{~V}_{1}, \mathrm{n}=17$ Panel $1 \mathrm{~V}_{2}$, potentially infectious). Figure $3 \mathrm{C}$ shows pools with $\mathrm{Cq} \geq 25(\mathrm{n}=32$ Panel $1 V_{1}, n=33$ Panel $1 V_{2}$ ) and Figure $3 \mathrm{D}$ pools with a $\mathrm{Cq}$ value between 25 and 30 which is the range where the RDT showed considerable differences $(n=23$ for both panels). The number of laboratories contributing to a result is presented in Table 2 .

For virus loads higher than $10^{6}$ genome copies per $\mathrm{mL}$ ( $\mathrm{Cq}<25)$, the sensitivity of 26 of the 31 RDT was higher than $80 \%$, indicating that these RDT would potentially identify infectious specimens with a probability of $80 \%$ (Figure 3A). For these RDT, the proficiency to detect those pools that contained culturable SARS-CoV-2 was even better, with values up to $100 \%$ (data not shown). For a virus load $\leq 10^{6}$ genome copies per $\mathrm{mL}(\mathrm{Cq} \geq 25)$, none of the evaluated tests could surpass a sensitivity of $80 \%$ (Figure 3B). In the Cq range between 25 and 30 , 10 of 31 RDT reached a sensitivity of $80 \%$ and higher, and five further RDT showed sensitivities only slightly 
Recommendation for panel usage to guarantee maximum comparability between different laboratories and points in time of validation, evaluation of the sensitivity of 31 rapid detection tests for SARS-CoV-2 diagnostics, Germany, September 2020April 2021

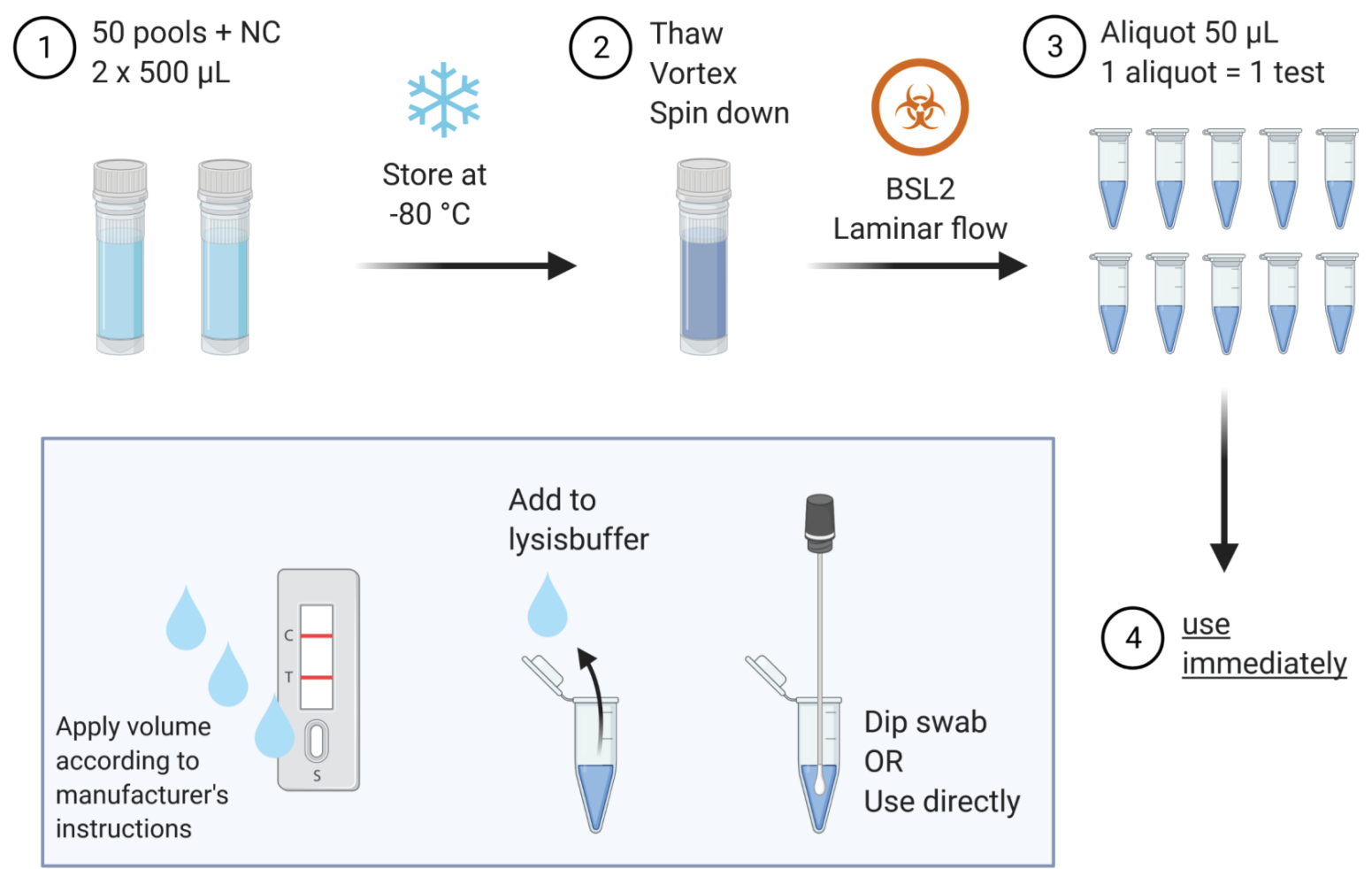

Created with BioRender.com

BSL: Biosafety level; NC: negative control

below $80 \%$ (Figure 3D). Finally, when the sensitivity to detect all 50 pools of the panel was determined, only four of 31 RDT passed a sensitivity of $80 \%$ or higher; two of those four RDT required a detection device for read-out. However, 10 of 31 RDT showed an overall sensitivity higher than $70 \%$ for the full panel (Figure $3 \mathrm{~A}$ ). A minimal detection rate of $75 \%$ for $\mathrm{Cq}<25$ was finally used as pass criterion in the comparative evaluation of 122 RDT as described in the tandem publication by Scheiblauer et al. [10]. Using a swab to transfer the volume of the pool material in the RDT test procedure led in the majority of evaluated RDT to reduced sensitivity. Hence, as described for the analytical sensitivity based on the RNA detection limit, clinical sensitivities were lower for most RDT when a swab was used.

Even if most of the RDT were analysed in two independent laboratories only, RDT \#1-5 and \#28-30 were evaluated by three to six laboratories with or without using swabs. As shown in Figures 2 and 3, there can be considerable variability for some tests, which is most probably due to the subjective interpretation of a positive test band; however, most results were very similar across laboratories and on different days.

\section{Discussion}

RDT are promising tools in the diagnostic portfolio of tools for the identification of SARS-CoV-2-infected individuals [14-16]. Since these tests, unlike PCR, do not use amplification of their target molecule, their analytical sensitivity is usually limited. Hence, the evaluation of RDT plays a major role in defining the suitable scenarios for the use of RDT. In contrast to PCR, where the specimen can be inactivated, RDT should be evaluated with clinical material that contains native viruses to mirror the diagnostic application as authentically as possible. However, the systematic comparison of various RDT in different laboratories at different times requires larger sample volumes and good storage stability.

Multiple sampling of naso- and/or oropharyngeal swabs is hampered by reproducibility. Even sampling the same patient with several swabs consecutively is likely to result in different viral loads per swab, which has to be controlled by real-time PCR and changes the test procedure. Because most RDT protocols require the clinical specimen to be sampled with a swab from which virus has to be eluted in the system-specific buffer, one swab cannot be used more than once without changing the protocol. Therefore, the decentralised evaluation of various RDT in different laboratories is difficult. So far, clinical samples with semi-quantified 
Analytical sensitivity of rapid diagnostic tests for SARS-CoV-2, expressed as 50\% detection probability, Germany, September 2020-April $2021(\mathrm{n}=31)$

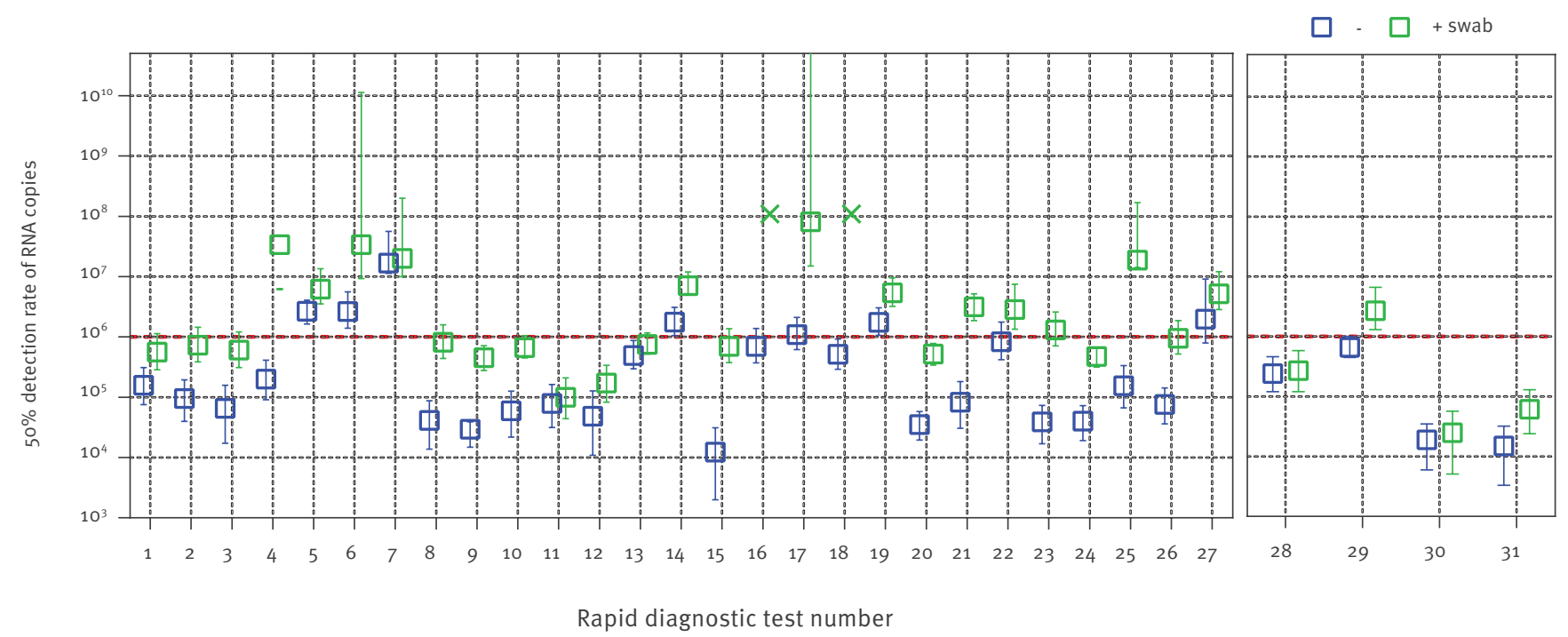

Cq: quantification cycle; RDT: rapid diagnostic test.

RDT \#28-31 require an additional device for reading out the result. Binary logistic regression was applied to calculate the analytical sensitivity from the 50 samples included in the panel. Open blue bars: virus-containing pools were directly subjected to the RDT without using a swab; open green squares: corresponding results when using a swab. Green crosses: tests that generated only one positive result for pool \#1 (Cq = 17.55; $1.1 \times 108$ RNA copies per $\mathrm{mL})$, while the other 49 pools remained negative. Calculation of the $50 \%$ detection probability was not possible for these RDT.

SARS-CoV-2 concentrations or virus propagated in cell culture have been used to validate RDT [17-19]. This can be done for a limited number of tests in a short period of time but is not suitable when numerous RDT have to be compared regularly in different laboratories.

Therefore, we established an evaluation panel that was used to determine the analytical and clinical sensitivity of RDT providing comparable results. The main basis of each pool were dry swabs in PBS originally used for PCR diagnostics. Since some of the swabs had been transported to the laboratory in viral transport medium, the final concentration of viral transport medium differed between pools. Although we confirmed in only 10 of the evaluated RDT that the presence of viral transport medium did not influence RDT sensitivity, we believe that this low medium content in the evaluation panel did not influence the test sensitivity in general, since pools with medium percentages between 10\% and $20 \%$ were distributed across the whole Panel $1 V_{1}$.

We determined the SARS-CoV-2 genome load by realtime $P C R$ in clinical specimens, and specimens with similar load were pooled, diluted in a background of negative swabs in PBS and the virus load quantified again. The established pools had a volume of $10 \mathrm{~mL}$ for both Panel $1 \mathrm{~V}_{1}$ and $1 \mathrm{~V}_{2}$ and covered a genome load from $1.1 \times 10^{9}$ to 400 genomes per $\mathrm{mL}$, which is the range of typical clinical specimens analysed in our laboratory. Even if the genome load does not reflect the number of virus particles directly, the RNA copy number was recently used to estimate the number of virus particles, reflecting the infectious potential of a specimen, and can correlate with the $\mathrm{N}$ protein concentration in clinical samples [20].

The fact that we used pools of up to 10 clinical specimens facilitates to some degree the compensation for potential variation between individual samples, for example varying ratios of genome copies vs number of viral particles or rather vs antigen concentration.

The pools of the panel showed results comparable to fresh clinical specimens with a similar SARS-CoV-2 genome load when selected RDT were used; freezing at -40 to $-80^{\circ} \mathrm{C}$ did not impact the detectability considerably. Nevertheless, as a trade-off for better reproducibility and comparability, the material used was not as fresh as in a clinical setting.

Usually, RDT use swabs that are subjected to the RDTspecific buffer before incubation of the test membrane. Our approach necessarily began with liquid specimens of $50 \mu \mathrm{L}$, which is intended for some RDT but not for all of them. However, with the intention to generate comparable and reproducible data, we accepted that some of the buffers were diluted by the fluid of the pools. Adding $50 \mu \mathrm{L}$ of pools directly to three randomly selected RDT without application of additional testspecific buffers, we observed that detectability was not impaired in comparison to the regular protocol, at least not in these RDT. 


\section{FIGURE 3}

Clinical sensitivities of rapid diagnostic tests for SARS-CoV-2 as determined by two to six laboratories using 50 pools from evaluation Panels 1V1 and 1V2, Germany, September 2020-April 2021 ( $=31$ )

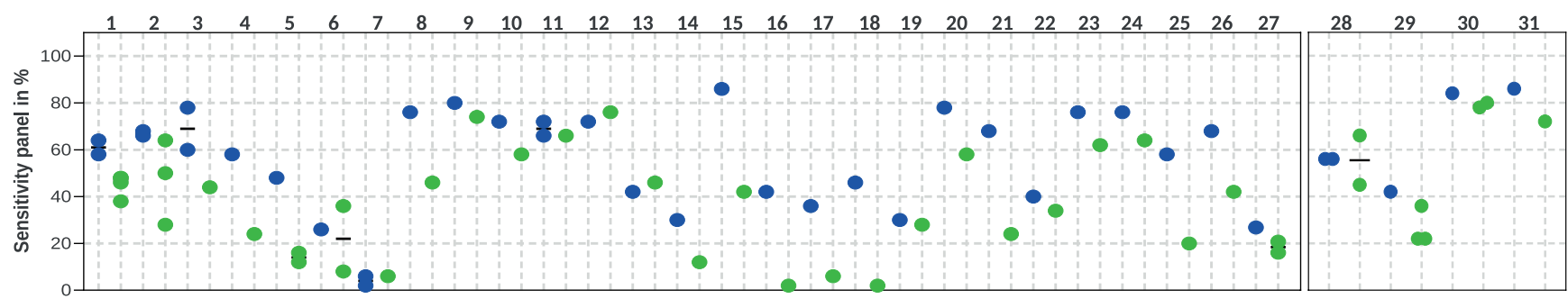

B.

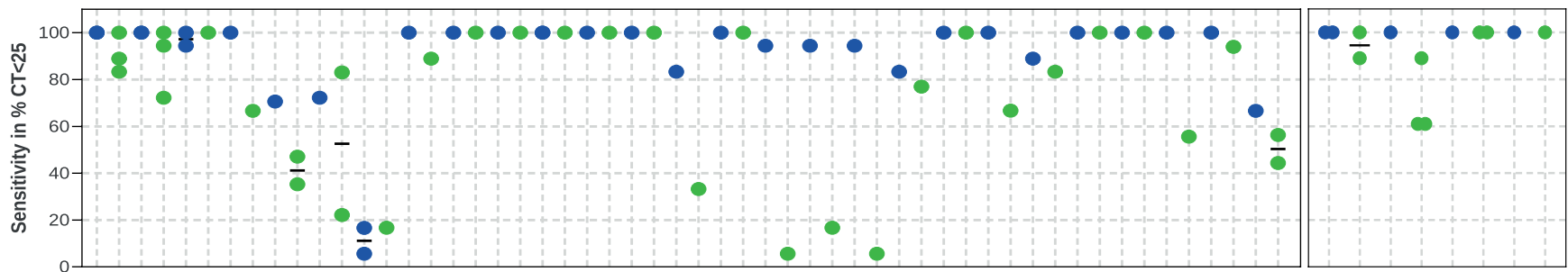

c.

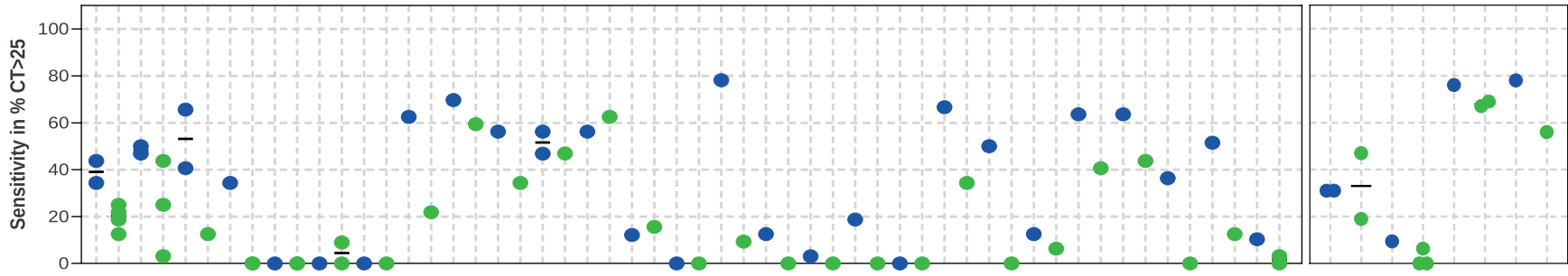

D.

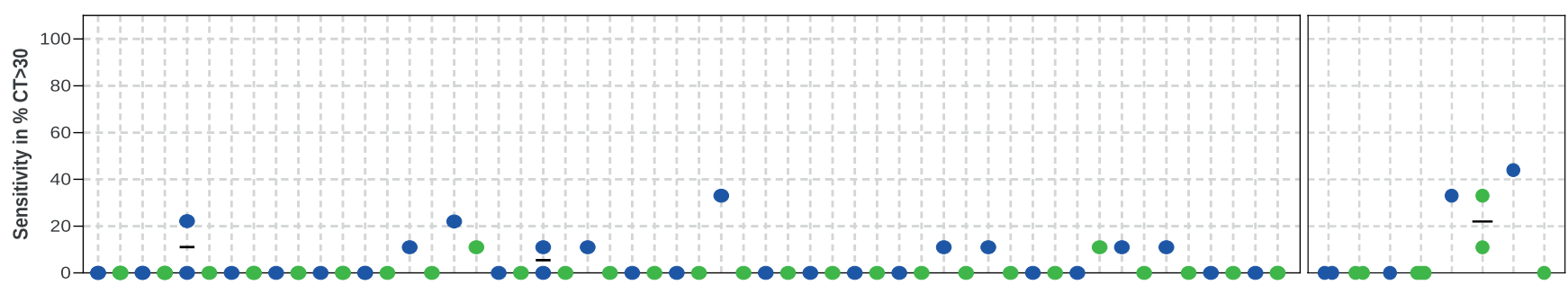

E.

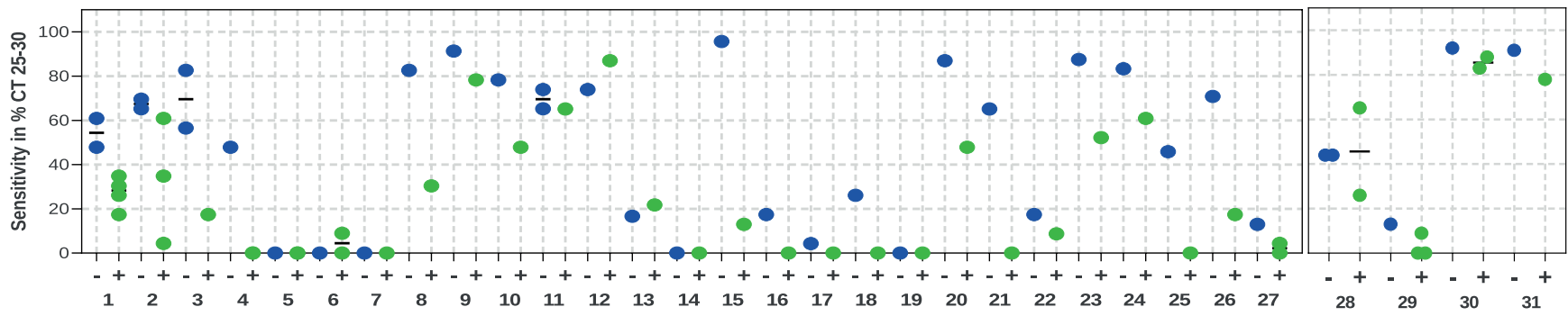

Rapid diagnostic test number

- swab + swab

Cq: quantification cycle; RDT: rapid diagnostic test.

Blue circles: direct application of the pool to the test buffer; green circles: RDT-specific swab used. Each circle represents the results of one laboratory (symbols may cover each other). RDT \#28-31 require an additional device for reading of result. 
The strategy of using liquid specimens comes with a further benefit, e.g. the option to cultivate the pools in cell culture, showing the infectivity of pools with a sufficient virus load. To our knowledge, this is one of the few studies that systematically evaluated several commercially available antigen RDT using standardised samples in comparison with real-time PCR as well as infectivity data from cell culture [21]. However, since all specimens included in a pool have been frozen and thawed at least once, the capability to grow in cell culture can be improved with fresh clinical specimens of a comparable virus amount [22].

Variability in the interpretation of RDT results observed in different laboratories has been described for diseases such as malaria and was significant for some RDT but, in general, highly comparable results were obtained [23]. Therefore, our results reflect the natural variance that can be expected when different users apply the RDT. It can be assumed that the interpretation of results is standardised better with RDT that require a device to read out the signal. Based on the number of RDT we have validated, we can confirm that read-out devices may help generate more reproducible results and reduce the inter-user variance.

The common RDT starts with the sampling that results in a swab containing material from the mucosa of the naso- or oropharynx. Then the swab is transferred into the RDT-specific buffer and subjected to the test membrane. Applying liquid evaluation specimens with known virus amounts does therefore not consider the impact of the swab on the result. The swab has to absorb liquids from the mucosa that potentially contain SARS-CoV-2 or can scrape off cellular material containing virus; probably it is a combination of both. Besides the problem that the swab does not absorb the specimen quantitatively, virus proteins can be retained by the swab and subsequently will not be subjected to the RDT, reducing the analytical sensitivity. While some of the RDT did not suffer considerably from using a swab before testing (\#12, \#13, \#28 and \#30), most of the tests lost sensitivity by a factor of 10 to 50 . This does not mean that the respective test device is inferior but rather that the swab is not efficient in absorbing and releasing SARS-CoV-2 from a liquid specimen. However, speculating that these swabs will come with the same drawbacks when used in clinical sampling, the loss of sensitivity can also occur. In a patient carrying for example $10^{6}$ genome copies in the nasopharynx, with RNA load used as a surrogate for viral particles, the sampling on the mucosa bears a risk of a false negative result in the RDT because of considerable loss of antigen in the swab. Further investigations of the efficiency of virus absorption and release from a swab will help interpret the risk of false negatives by sampling. Besides, further specimen types such as saliva are under investigation for their use in RDT. Along these lines, we are currently further investigating the role of swab types on the uptake and release of virus material (study ongoing).

Finally, our study did not address the specificity of an RDT and cannot assess the risk of false positive results. At least all assessed RDT were negative for the negative control. However, recent studies show that high specificity is reached by most of the RDT evaluated [24]. In addition to SARS-CoV-2-positive specimens, the next version of our evaluation panel will also include SARS-CoV-2-negative specimens positive for other respiratory viruses.

\section{Conclusion}

The sensitivity of the 31 RDT evaluated in this study varied extensively and depended largely on the virus load in the respective specimen. While four RDT showed a sensitivity of $>80 \%$ over the whole range of virus loads investigated, 26 RDT had a sensitivity of $>80 \%$ for potentially infectious specimens, indicating that sensitive RDT can be used to identify contagious individuals in various settings, but not to identify infected individuals with lower virus loads. Our results are in agreement with several other studies not using a standardised evaluation panel [25], indicating the applicability of the described panel for RDT evaluation. The minimal performance characteristics of an RDT have recently been discussed by the WHO to be at least $80 \%$ for symptomatic patients [15]. Considering that virus loads vary during the time course of infection in an individual and between individuals, the sensitivity of an RDT should rather be attributed to a certain virus load rather than the time after onset of symptoms or after a qualitative $P C R$ result.

\section{Acknowledgements}

The authors are grateful to Ursula Erikli for copy-editing. This work was funded by the Ministry of Health, Germany (Maßnahmepaket 1\&2).

\section{Conflict of interest}

None declared.

Authors' contributions

Andreas Puyskens, Eva Krause, Janine Michel and Marica Grossegesse designed the study, established the evaluation panel and performed the RDT with Roman Valusenko. Daniel Bourquain performed cell culture experiments. Micha Nübling and Heinrich Scheiblauer characterised the panel and evaluated several RDT. Victor M. Corman, Christian Drosten, Katrin Zwirglmaier, Roman Wölfel, Constanze Lange, Jan Kramer, Johannes Friesen, Ralf Ignatius, Michael Müller, Jonas Schmidt-Chanasit and Petra Emmerich provided data for varying numbers of RDT with the evaluation panel. Lars Schaade and Andreas Nitsche conceptualised the study, analysed the data and wrote the manuscript. 


\section{References}

1. Nalla AK, Casto AM, Huang MW, Perchetti GA, Sampoleo R, Shrestha L, et al. Comparative performance of SARS-CoV-2 detection assays using seven different primer-probe sets and one assay kit. J Clin Microbiol. 2020;58(6):e00557-20. https:// doi.org/10.1128/JCM.00557-20 PMID: 32269100

2. Mackay IM, Arden KE, Nitsche A. Real-time fluorescent PCR techniques to study microbial-host interactions. In: Savidge T, Pothulakis C, editors. Microbial Imaging. Methods in Microbiology vol. 34, Amsterdam: Elsevier/ Academic Press; 2004, p.: 255-330. https://doi.org/10.1016/ S0580-9517(04)34010-9

3. Peeling RW, McNerney R. Emerging technologies in pointof-care molecular diagnostics for resource-limited settings. Expert Rev Mol Diagn. 2014;14(5):525-34. https://doi.org/10.15 86/14737159.2014.915748 PMID: 24784765

4. Strömer A, Rose R, Schäfer M, Schön F, Vollersen A, Lorentz T, et al. Performance of a point-of-care test for the rapid detection of SARS-CoV-2 antigen. Microorganisms. 2020;9(1):58. https:// doi.org/10.3390/microorganisms9010058 PMID: 33379279

5. Jacobs J, Kühne V, Lunguya O, Affolabi D, Hardy L, Vandenberg 0. Implementing COVID-19 (SARS-CoV-2) rapid diagnostic tests in Sub-Saharan Africa: a review. Front Med (Lausanne). 2020;7:557797. https://doi.org/10.3389/fmed.2020.557797 PMID: 33195307

6. Kozel TR, Burnham-Marusich AR. Point-of-care testing for infectious diseases: past, present, and future. J Clin Microbiol. 2017;55(8):2313-20. https://doi.org/10.1128/JCM.00476-17 PMID: 28539345

7. Berzosa P, de Lucio A, Romay-Barja M, Herrador Z, González V, García L, et al. Comparison of three diagnostic methods (microscopy, RDT, and PCR) for the detection of malaria parasites in representative samples from Equatorial Guinea. Malar J. 2018;17(1):333. https://doi.org/10.1186/s12936-0182481-4 PMID: 30223852

8. FIND evaluation of SARS-CoV-2 antigen (Ag) detecting tests. Geneva: FIND. [Accessed: 20 Oct 2021]. Available from: https:// www.finddx.org/sarscov2-eval-antigen

9. Michel J, Neumann M, Krause E, Rinner T, Muzeniek T, Grossegesse $M$, et al. Resource-efficient internally controlled in-house real-time PCR detection of SARS-CoV-2. Virol J. 2021;18(1):110. https://doi.org/10.1186/s12985-021-01559-3 PMID: 34078394

10. Scheiblauer H, Filomena A, Nitsche A, Puyskens A, Corman VM, Drosten C, et al. Comparative sensitivity evaluation for 122 CE-marked SARS-CoV-2 antigen rapid tests. Euro Surveill. 2021;26(44):2100441.

11. Wölfel R, Corman VM, Guggemos W, Seilmaier M, Zange S, Müller MA, et al. Virological assessment of hospitalized patients with COVID-2019. Nature. 2020;581(7809):465-9. https://doi.org/10.1038/s41586-020-2196-x PMID: 32235945

12. Kim M-C, Cui C, Shin K-R, Bae J-Y, Kweon O-J, Lee M-K, et al. Duration of culturable SARS-CoV-2 in hospitalized patients with Covid-19. N Engl J Med. 2021;384(7):671-3. https://doi. org/10.1056/NEJMc2027040 PMID: 33503337

13. van Kampen JJA, van de Vijver DAMC, Fraaij PLA, Haagmans BL, Lamers MM, Okba N, et al. Duration and key determinants of infectious virus shedding in hospitalized patients with coronavirus disease-2019 (COVID-19). Nat Commun. 2021;12(1):267. https://doi.org/10.1038/s41467-020-20568-4 PMID: 33431879

14. La Scola B, Le Bideau M, Andreani J, Hoang VT, Grimaldier C, Colson P, et al. Viral RNA load as determined by cell culture as a management tool for discharge of SARS-CoV-2 patients from infectious disease wards. Eur J Clin Microbiol Infect Dis. 2020;39(6):1059-61. https://doi.org/10.1007/s10096-02003913-9 PMID: 32342252

15. World Health Organization (WHO). COVID-19 target product profiles for priority diagnostics to support response to the COVID-19 pandemic v.1.0. Geneva: WHO; 2020. Available from: https://www.who.int/publications/m/item/covid-19-targetproduct-profiles-for-priority-diagnostics-to-support-responseto-the-covid-19-pandemic-v.0.1

16. Berger A, Nsoga MTN, Perez-Rodriguez FJ, Aad YA, SattonnetRoche P, Gayet-Ageron A, et al. Diagnostic accuracy of two commercial SARS-CoV-2 antigen-detecting rapid tests at the point of care in community-based testing centers. PLoS One. 2021;16(3):e0248921. https://doi.org/10.1371/journal. pone.0248921 PMID: 33788882

17. Mboumba Bouassa RS, Veyer D, Péré H, Bélec L. Analytical performances of the point-of-care SIENNA ${ }^{\mathrm{TM}}$ COVID-19 Antigen Rapid Test for the detection of SARS-CoV-2 nucleocapsid protein in nasopharyngeal swabs: A prospective evaluation during the COVID-19 second wave in France. Int J Infect Dis. 2021;106:8-12. https://doi.org/10.1016/j.ijid.2021.03.051 PMID: 33746093
18. Olearo F, Nörz D, Heinrich F, Sutter JP, Roedl K, Schultze A, et al. Handling and accuracy of four rapid antigen tests for the diagnosis of SARS-CoV-2 compared to RT-qPCR. J Clin Virol. 2021;137:104782. https://doi.org/10.1016/j.jcv.2021.104782 PMID: 33711691

19. Corman VM, Haage VC, Bleicker T, Schmidt ML, Mühlemann $B$, Zuchowski M, et al. Comparison of seven commercial SARS-CoV-2 rapid point-of-care antigen tests: a single-centre laboratory evaluation study. Lancet Microbe. 2021;2(7):e311 9. https://doi.org/10.1016/S2666-5247(21)00056-2 PMID: 33846704

20. Pollock NR, Savage TI, Wardell H, Lee R, Mathew A, Stengelin $M$, et al. Correlation of SARS-CoV-2 nucleocapsid antigen and RNA concentrations in nasopharyngeal samples from children and adults using an ultrasensitive and quantitative antigen assay. Preprint. MedRxiv. 2020:11.10.20227371. https://doi.or $\mathrm{g} / 10.1101 / 2020.11 .10 .20227371$

21. Kohmer N, Toptan T, Pallas C, Karaca O, Pfeiffer A, Westhaus $\mathrm{S}$, et al. The comparative clinical performance of four SARSCoV-2 rapid antigen tests and their correlation to infectivity in vitro. J Clin Med. 2021;10(2):328. https://doi.org/10.3390/ jcm10020328 PMID: 33477365

22. Basile K, McPhie K, Carter I, Alderson S, Rahman H, Donovan L, et al. Cell-based culture of SARS-CoV-2 informs infectivity and safe de-isolation assessments in patients during COVID-19. Clin Infect Dis. 2020; ciaa1579. https://doi.org/10.1093/cid/ ciaa1579 PMID: 33098412

23. Boyce MR, Menya D, Turner EL, Laktabai J, PrudhommeO'Meara W. Evaluation of malaria rapid diagnostic test (RDT) use by community health workers: a longitudinal study in western Kenya. Malar J. 2018;17(1):206. https://doi. org/10.1186/s12936-018-2358-6 PMID: 29776359

24. Baro B, Rodo P, Ouchi D, Bordoy AE, Saya Amaro EN, Salsench $\mathrm{SV}$, et al. Performance characteristics of five antigen-detecting rapid diagnostic test (Ag-RDT) for SARS-CoV-2 asymptomatic infection: a head-to-head benchmark comparison. J Infect. 2021;82(6):269-75. https://doi.org/10.1016/j.jinf.2021.04.009 PMID: 33882299

25. Dinnes J, Deeks IJ, Adriano A, Berhane S, Davenport C, Dittrich $S$, et al. Rapid, point-of-care antigen and molecular-based tests for diagnosis of SARS-CoV-2 infection. Cochrane Database Syst Rev. 2020;8:CD013705. https://doi.org/10.1002/14651858. CD013705 PMID: 32845525

\section{License, supplementary material and copyright}

This is an open-access article distributed under the terms of the Creative Commons Attribution (CC BY 4.0) Licence. You may share and adapt the material, but must give appropriate credit to the source, provide a link to the licence and indicate if changes were made.

Any supplementary material referenced in the article can be found in the online version.

This article is copyright of the authors or their affiliated institutions, 2021. 